\title{
Desarrollo de un sistema para el registro de variables termodinámicas en un equipo de enfriamiento
}

\author{
Development of a System for the Recording of Thermodynamic Variables in a \\ Refrigeration Equipment
}

\author{
Federico Roffé, Guillermo O. Lombardero, Guillermo R. Sánchez \\ Departamento de Fisica - Departamento de Ingeniería - UNNE-FACENA \\ Campus Av. Libertad 5470 Corrientes, Argentina \\ imit-bec-frodcomunidad.unne.edu.ar
}

Recibido: 12/02/21; Aceptado: 30/04/21

\begin{abstract}
This project consisted of the design, implementation and calibration of a system for recording variables of the operation of a cooling equipment. It was sought to have parameters for the analysis of efficiency and optimal establishment of the work cycle. The measurement system was implemented in a refrigeration circuit of the Thermodynamics Laboratory of the FACENA-UNNE, considering normal working conditions.
\end{abstract}

Keywords: cooling equipment, refrigeration system, duty cycle.

Resumen- Este proyecto consistió en el diseño, implementación y calibración de un sistema de registro de variables del funcionamiento de un equipo de refrigeración. Se buscó contar con parámetros para el análisis de eficiencia y establecimiento óptimo del ciclo de trabajo. El sistema de medición se implementó en un circuito de refrigeración del Laboratorio de Termodinámica de la FACENA-UNNE, teniendo en cuenta las condiciones normales de trabajo.

Palabras clave: equipo de enfriamiento, sistema de refrigeración, ciclo de trabajo.

\section{INTRODUCCIÓN}

La refrigeración es un proceso que consiste en bajar o mantener el nivel térmico de un cuerpo o un espacio. Cuando hablamos de frío nos referimos a una menor cantidad de calor que posee un cuerpo con respecto al ambiente que lo contiene y que debe hablarse de mayor o menor cantidad de calor o de mayor o menor nivel térmico (nivel que se mide con la temperatura). Refrigerar es un proceso termodinámico en el que se extrae calor del objeto considerado (reduciendo su nivel térmico), y se lleva a otro, que sea capaz de admitir esa energía térmica sin problemas. Los fluidos utilizados para llevar la energía calorífica de un espacio a otro son llamados refrigerantes [1] y dependiendo de los fines, la refrigeración puede hacerse de varios modos [2].

El método normalmente utilizado para el enfriamiento de espacios cerrados se consigue mediante los procesos de compresión y de absorción. El tratamiento por compresión es de estos dos el más frecuente, puesto que el procedimiento por absorción solo se suele utilizar cuando hay una fuente de calor residual. La refrigeración por compresión es un método que consiste en forzar mecánicamente la circulación de un refrigerante en un circuito cerrado dividido en dos zonas, una de alta y otra de baja presión, con el propósito de que el fluido absorba calor del ambiente en el evaporador (baja presión) y lo ceda en el condensador (alta presión). Se basa en las propiedades de ciertos refrigerantes, siendo la principal su temperatura de vaporización que a presión atmosférica es extremadamente baja. Los refrigerantes utilizados comúnmente, tienen temperaturas de ebullición en condiciones normales, alrededor de $-40^{\circ} \mathrm{C}$.

El circuito de refrigeración corresponde a un arreglo mecánico basado en los principios de la termodinámica y mecánica de fluidos diseñado para transferir energía térmica entre dos focos (sistemas termodinámicos capaces de intercambiar cualquier cantidad de calor sin que cambien sus propiedades), desplazando la energía térmica contenida en uno de sus focos a fin de obtener una menor temperatura en este. Estos focos intercambian energía (calor, trabajo) con el exterior, pero nunca materia (su masa permanece intacta). Este cometido se lleva a cabo forzando la circulación de un fluido refrigerante por el interior de un circuito cerrado o semicerrado de tuberías e intercambiadores de calor. La circulación de este fluido refrigerante se realiza a través de máquinas como compresores $\mathrm{y} / \mathrm{o}$ bombas, conforme la naturaleza y estado del refrigerante [3].

Un diagrama P-h, o diagrama de Mollier para presión entalpía, como se puede observar en Fig. 1, es la representación gráfica en una carta semilogarítmica en el plano presión/entalpía de los estados posibles de un compuesto químico, especialmente para los gases refrigerantes, y es en ella donde se trazan y suelen estudiar los distintos sistemas frigoríficos de refrigeración por compresión. Por su parte, cada refrigerante tiene su propio diagrama P-h con particularidades que lo hacen más o menos adecuado a cada aplicación frigorífica y propiedades exclusivas como relación temperatura/presión tanto de saturación, mezcla, efecto refrigerante, temperatura de descarga del compresor en función de la entropía, entre otros. 


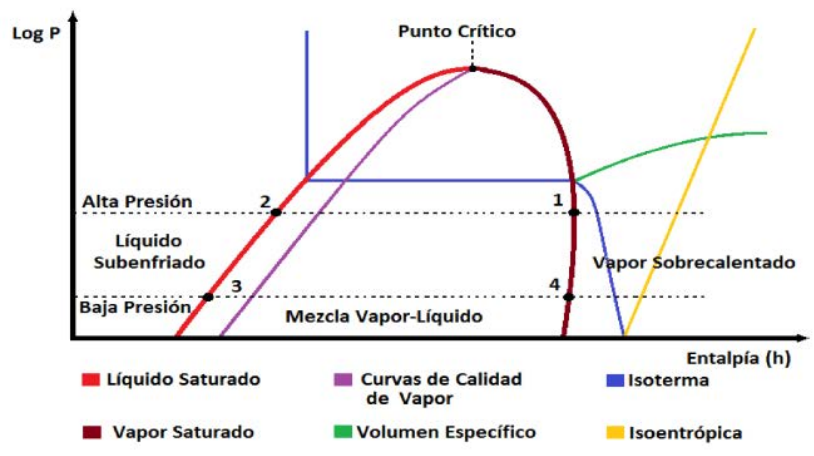

Fig. 1. Diagrama de Mollier simplificado para un ciclo cerrado.

Una de las ventajas del diagrama P-h es la facilidad con que se pueden realizar los cálculos de sistemas frigoríficos y selección de componentes como evaporadores, condensadores, compresores, dispositivos de expansión, tuberías y accesorios, así como trazar todo tipo de sistemas frigoríficos de una etapa, compresión múltiple, sistemas en cascada, sistemas con recirculado por bomba y otros.

Supuesto un refrigerante que cumple con todas estas características, el proceso en un circuito frigorífico se lleva a cabo elevando su presión y temperatura, mediante un compresor, hasta alcanzar la presión de condensación (punto 1 en Fig. 1). En esas condiciones el fluido atraviesa el condensador mientras intercambia calor con el medio exterior. Como consecuencia de la cesión de calor se produce la condensación del fluido, que sale del condensador y alcanza la válvula de expansión totalmente en estado líquido (punto 2 en Fig. 1). Esta parte del proceso se puede considerar isotérmica, ya que no varía la temperatura durante el cambio de estado.

El dispositivo de expansión provoca una caída repentina de la presión y la temperatura sin intercambio de calor, por lo que esta parte del proceso se puede considerar como una transformación adiabática o isoentálpica (punto 3 en Fig. 1). El fluido todavía en estado líquido y a la presión de vaporización circula por el evaporador, del cual se absorbe la energía térmica correspondiente al calor latente de vaporización, de forma tal que el fluido sale del evaporador completamente en estado de vapor. La transformación se puede considerar isotérmica por la misma razón que se dio en el condensador. A continuación, el vapor es aspirado por el compresor para iniciar de nuevo el ciclo (punto 4 en Fig. 1).

En el ciclo de refrigeración ideal, en los balances de energía del equipo, se desprecia cualquier pérdida o ganancia de calor en las tuberías, considerando que los únicos intercambios de calor que se producen en el sistema ocurren en el evaporador y en el condensador. Sin embargo, en el ciclo real el fluido refrigerante sufre una ligera caída de presión y temperatura debido a las perdidas por fricción, sobre todo en evaporador, condensador y en las restricciones de las válvulas de admisión y de escape. Esto hace que el ciclo real resulte ligeramente distorsionado respecto del ciclo ideal.

Por otro lado, el Protocolo de Kyoto hace necesario un aumento del rigor en la aplicación y la investigación de nuevas técnicas ya que la mayoría de los sistemas de refrigeración y de aire acondicionado usan una considerable cantidad de energía y por lo tanto contribuyen ya sea directa o indirectamente al calentamiento global [4].

Considerando lo expuesto anteriormente, surge la necesidad de desarrollar un sistema que permita analizar las variables termodinámicas de un medio de enfriamiento, para determinar el funcionamiento y ciclo de trabajo de un circuito de refrigeración, permitiendo un estudio de las magnitudes físicas y un análisis detallado de los estados.

\section{MATERIALES Y MÉTODOS}

El trabajo se inició con una investigación bibliográfica, la cual se centró en la búsqueda y recopilación de información sobre circuitos de refrigeración, los sensores a utilizar, el sistema a implementar y un medio para la adquisición y tabulación de datos basados en la plataforma Arduino. Luego de un análisis exhaustivo se pudo concluir en la necesidad de definir variables de contorno. El criterio que se tomó para la elección de las variables del sistema de medición fue el de lograr establecer un ciclo de trabajo mediante un diagrama de Mollier y calcular su coeficiente de desempeño (COPr).

Una vez que se definieron cuáles eran las variables se procedió a diseñar el sistema que permitió la medición y adquisición de información, así como su almacenamiento para el tratamiento de los datos y sus análisis posteriores. Las variables termodinámicas para el diseño del sistema de medición fueron, la temperatura del gas refrigerante en diferentes puntos del circuito de refrigeración, la humedad relativa y temperatura a la entrada y salida del aire ambiente interior del evaporador, y el consumo de energía del equipo. La elección de los sensores se basó en la necesidad de las magnitudes a medir, el rango de medición y su disponibilidad comercial.

\section{A. Calibración de los sensores}

En primer lugar, luego de adquirir los sensores, se practicaron pruebas a modo de testeo usando una placa Arduino basada en el Microcontrolador Atmega 2560.

Una vez conectados estos sensores a la placa se elaboró un programa básico en la plataforma Arduino IDE (sketch) para ir probando uno a uno estos, así como observar y analizar qué datos se obtenían, y si eran consistentes con las mediciones.

Uno de los problemas al momento de tomar mediciones era el ruido incorporado en las señales, ya que éste las hacia variar en un cierto rango, es decir, tomando muestras consecutivas en iguales condiciones, los datos eran diferentes. Para solucionar este inconveniente se procedió a establecer un método de medición que anule lo máximo posible estas variaciones. El método consistió en trabajar sobre el programa para disminuir la incidencia del ruido. Esta corrección se logró tomando un número finito de muestras y realizando su promedio estadístico.

Otro problema que surgió fue el de que los sensores arrojaron valores de medición diferentes, siendo que dichas mediciones se realizaban en condiciones ambientales iguales. Estos valores eran próximos entre sí, pero no lo suficiente para tomarlos como verdaderos. Las discrepancias entre los valores medidos se debieron a que cada sensor posee un pequeño corrimiento del cero de medición (offset), y cada uno de estos corrimientos es diferente para cada sensor.

Para corregir esta cuestión se procedió a efectuar una calibración de los sensores mediante un parámetro de referencia. En el caso de la medición de temperatura, por ejemplo, se aplicó la calibración utilizando como parámetro patrón una resistencia de platino, aprovechando su exactitud y fiabilidad.

La calibración consistió en poner todos los sensores en un tubo de ensayo de vidrio aislando lo máximo posible el 
ingreso de aire dentro de este, luego este tubo se sumergió en un recipiente con hielo y agua destilada para establecer un equilibrio térmico entre todos los sensores y marcar la temperatura de $0^{\circ}$ Celsius. Posteriormente se fue incrementando la temperatura de la mezcla del recipiente con agua caliente y batiendo por momentos todo el sistema, y así se fueron encontrando en diferentes periodos de tiempo los incrementos de temperatura hasta llegar a $\operatorname{los} 50^{\circ} \mathrm{C}$.

Para la siguiente etapa se puso el tubo en agua hirviendo para lograr el equilibrio térmico a $\operatorname{los} 100^{\circ} \mathrm{C}$, y luego se fue disminuyendo la temperatura con agua fría destilada mezclada con hielo, batiendo todo el conjunto por momentos, hasta que se alcanzó a los $50^{\circ} \mathrm{C}$.

Concluido este procedimiento se logró establecer los valores parámetros para las mediciones de los sensores. Con estos datos se corrigió el corrimiento sobre cada uno y se equilibró todas las medidas de temperatura.

\section{B. Mediciones}

Para medir físicamente el calentamiento de un equipo de refrigeración se deben seguir los siguientes pasos, se necesita conocer la temperatura a la que llega el gas al compresor y la temperatura a la que el refrigerante ha estado cambiando de estado.

La temperatura en la aspiración del compresor se puede medir con un contacto en la superficie exterior del tubo de esa zona, esto es válido porque el refrigerante apenas absorbe energía en este tramo final, dándose como iguales el valor de temperatura del tubo y la temperatura del refrigerante que por él circula.

La temperatura del cambio de estado es difícil de medir, porque se necesita un elemento de medida que directamente ofrezca la lectura de la temperatura que tiene el refrigerante que circula por el interior de la tubería, en este caso no se puede hacer la medición en la superficie del tubo por el que circula, porque en esta zona el refrigerante está adquiriendo energía para cambiar de estado de forma violenta, si se hace la lectura de temperatura igual que antes, el contacto nos daría la temperatura a la que el refrigerante es capaz de enfriar nuestro sensor pero no ofrecería la temperatura a la que se encuentra el refrigerante realmente. La única opción es obtener el valor de temperatura partiendo del valor de presión en el circuito, recordando que a cada valor de este de un refrigerante le corresponde un valor de temperatura. En este caso se puede obtener la lectura de presión del evaporador con un manómetro y mediante la tabla de características del gas obtener la temperatura correspondiente.

Una vez que se han obtenido ambos valores de temperatura solamente se tiene que restar a la temperatura de aspiración la temperatura de cambio de estado y el valor obtenido tiene que estar comprendido entre 5 y $8^{\circ} \mathrm{C}$.

Para garantizar que el refrigerante llega a la aspiración del compresor en las condiciones óptimas de recalentamiento se puede actuar sobre distintos elementos que de forma directa o indirecta modifican el valor de recalentamiento, las actuaciones que se pueden realizar para variar el grado de recalentamiento y en qué dirección se las tendría que llevar a cabo. Estas acciones se resumen en la siguiente tabla:
TABLA I

Acciones para Modificar el Recalentamiento

\begin{tabular}{|l|l|}
\hline $\begin{array}{l}\text { Regular el paso de } \\
\text { aire }\end{array}$ & $\begin{array}{l}\text { Falta de aire produce un recalentamiento } \\
\text { bajo. } \\
\text { Exceso de aire produce un recalentamiento } \\
\text { alto. }\end{array}$ \\
\hline $\begin{array}{l}\text { Modificar la } \\
\text { cantidad de carga } \\
\text { del equipo }\end{array}$ & $\begin{array}{l}\text { Exceso de carga tiende a tener un } \\
\text { recalentamiento bajo o incluso nulo. } \\
\text { Falta de carga tiende a tener un } \\
\text { recalentamiento muy alto. }\end{array}$ \\
\hline $\begin{array}{l}\text { Regular el paso de } \\
\text { refrigerante con el } \\
\text { expansor }\end{array}$ & $\begin{array}{l}\text { Expansor muy abierto tiende a tener un } \\
\text { recalentamiento muy bajo. } \\
\text { Expansor muy cerrado tiende a tener un } \\
\text { recalentamiento muy alto. }\end{array}$ \\
\hline $\begin{array}{l}\text { La dimensión del } \\
\text { evaporador tiene } \\
\text { que ser acorde al } \\
\text { equipo }\end{array}$ & $\begin{array}{l}\text { Evaporador grande tiende a tener un } \\
\text { recalentamiento muy grande. } \\
\text { Evaporador pequeño tiende a tener un } \\
\text { recalentamiento pequeño. }\end{array}$ \\
\hline
\end{tabular}

El estado en el que el refrigerante llega al expansor es capaz de condicionar el funcionamiento del equipo tanto como para llegar al extremo de que nuestro equipo frigorífico no sea capaz de funcionar correctamente, aunque en menor medida que el grado de recalentamiento las condiciones que el refrigerante tiene que cumplir son bastante estrictas para que se garantice el buen funcionamiento del circuito frigorífico en general. Si estas características no se cumplen en la mayoría de los casos el único problema es que nuestro equipo rendirá mal, lo que vendría a decirnos que consumiría mucha energía para producir una pequeña cantidad de frío, además podría llegar a darse, en algún caso muy extremo la completa falta de producción de frío e incluso la avería del circuito frigorífico si no está regulado correctamente. El correcto estado del refrigerante en la entrada del expansor se cuantifica en función de la cantidad de líquido (tapón de líquido) formado antes de la expansión y del espacio de tiempo que el refrigerante espera su turno hasta poder atravesarlo.

La formación de un tapón de líquido en la entrada del expansor se consigue por la completa transformación del refrigerante a lo largo del recorrido del condensador, la finalidad es que el líquido que tiene muy poca capacidad para comprimirse al encontrarse al expansor (que es una obstrucción casi completa en el circuito frigorífico), quede retenido pues no puede pasar toda la cantidad de líquido que llega por un orificio tan pequeño. Al acumularse se termina formando una retención en la circulación del refrigerante. Esta retención formada por el refrigerante a la espera de expansionarse es la encargada de mantener el desequilibrio de presiones entre las dos partes del circuito, pues el expansor por sí solo no tiene capacidad ni posibilidad de generar el desequilibrio de presiones que es imprescindible para la correcta transformación del refrigerante a lo largo del circuito frigorífico (debe recordarse que se necesita bajas presiones en el evaporador y altas en el condensador para facilitar la evaporación y la condensación respectivamente), el tapón de líquido del que se hace mención tiene que estar dentro de unos parámetros no siendo válido un valor de tapón cualquiera.

El efecto de la falta de tapón se traduce en la falta de esta formación de líquido antes del expansor y esto puede producir una serie de efectos sobre el circuito frigorífico. Conociéndose lo que ocurre se puede valorar la importancia que realmente tiene la falta del tapón de líquido. 
La falta de desequilibrio de presiones entre condensador y evaporador se da cuando por alguna razón al final del condensador no se ha conseguido que el refrigerante sea completamente líquido, sino que se sigue tratando de refrigerante en forma de vapor más o menos saturado, lo que ocurriría seria que el refrigerante al encontrase en estado vapor, tiene mucha facilidad para comprimirse, aunque encuentre una obstrucción casi completa en el circuito como de hecho es el expansor, el refrigerante en forma de vapor al pasar por el expansor se comprimiría y aumentaría su velocidad al pasar por el estrechamiento, para producir la menor retención posible, produciéndose simplemente una pérdida de carga debida al rozamiento del vapor con el expansor, que es insuficiente para retener el paso del resto del refrigerante. Al no poder retener al resto del refrigerante, las presiones entre el condensador y el evaporador serían prácticamente iguales, y como se expuso anteriormente, las condiciones de presión son el elemento más importante para que se produzcan los cambios de estado en las diversas zonas del circuito frigorífico.

La Falta de refrigeración del compresor, se debe que al no existir condiciones de presión propicias para el cambio de estado el refrigerante en el condensador no se ve forzado a cambiar de estado, lo que permitiría que el refrigerante no ceda energía llegando al evaporador en estado gas caliente, este gas caliente es imposible que refrigere el compresor lo que podría producir la rotura del compresor por exceso de calentamiento si este no está dotado de elementos de protección.

El efecto del exceso de tapón de líquido antes del expansor puede producir una serie de efectos sobre el circuito frigorífico. Las altas presiones en el condensador se darían como efecto más visible de un exceso de líquido retenido antes del expansor, suponiendo que por alguna razón se tiene un tapón de líquido excesivo, este tapón indicará que se tiene mucho refrigerante esperando su turno para pasar por el expansor. Si la cantidad de líquido es muy grande no cabrá en el pequeño tramo de tubería que se tiene desde la salida del condensador al expansor, por tanto, el único lugar donde se podrá ir alojando todo el refrigerante en su espera hasta llegar al expansor, serán las ultimas tuberías del condensador.

Cuando se llenan de líquido las tuberías se las está anulando para su primordial finalidad que es servir de intercambio con el exterior para que los vapores que salen del compresor lleguen a condensarse, al inutilizar las tuberías se estaría haciendo el condensador más pequeño, con lo que el refrigerante para conseguir el mismo nivel de intercambio no tiene otra opción que elevar su temperatura o lo que es lo mismo aumentar su presión. Cuando se tiene presiones muy altas en la descarga al compresor le cuesta mucho más el trasladar el refrigerante del evaporador al condensador, y esto se traduce a su vez en un aumento de consumo para mover la misma cantidad de refrigerante, además si las presiones en el condensador son muy altas se estaría aumentando las posibilidades de que el compresor se pueda llegar a agarrotar, por no poder alcanzar presiones tan altas.

La dificultad de conseguir una correcta regulación en un circuito frigorífico estriba en que por un lado se necesita que el refrigerante llegue al expansor en estado líquido, pero por otro la cantidad de líquido no debe de ser muy grande, la solución que se toma para poder cumplir ambas necesidades es la de llegar a un punto intermedio de subenfriamiento del refrigerante en la llegada al expansor.

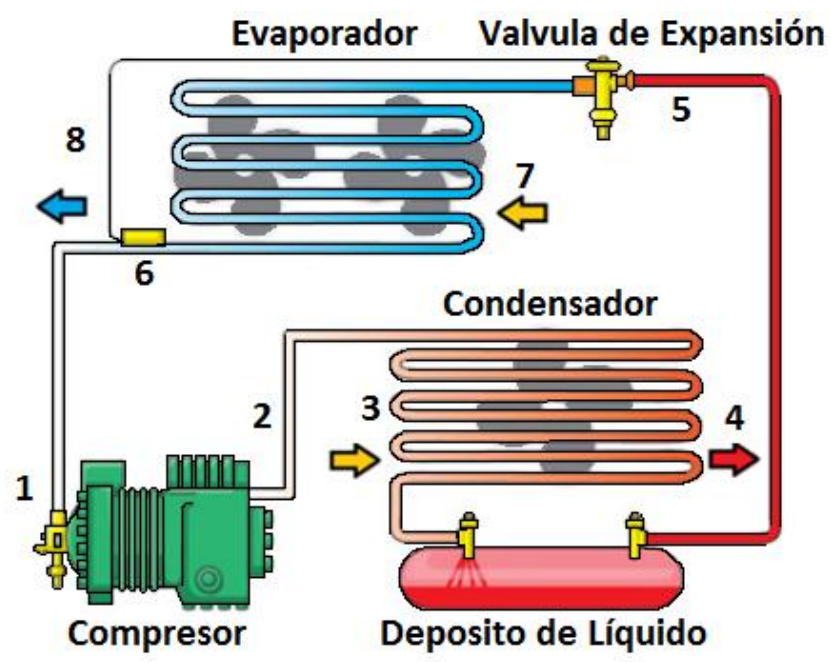

Fig. 2. Disposición determinada para la ubicación de los sensores a través del circuito de refrigeración. Los numerados del 1 al 6 son LM35 y el 7 y 8 los DHT22.

El punto intermedio debe de garantizar el mejor rendimiento posible, con la seguridad de que la cantidad de líquido que se tiene a la salida del condensador es una cantidad correcta, a este ajuste de enfriamiento posterior al cambio de estado se lo denomina grado de subenfriamiento. El grado de subenfriamiento que se ha establecido como óptimo para los equipos de aire acondicionado debe de estar comprendido entre 5 y $8^{\circ} \mathrm{C}$ menos que la temperatura a la que el refrigerante cambió de estado a lo largo del condensador.

Para medir físicamente el grado de subenfriamiento en cuestión es necesario conocer la temperatura a la que el refrigerante líquido llega al expansor y la temperatura a la que el refrigerante ha estado cambiando de estado.

Las maquinas térmicas, incluso bajo condiciones ideales deben rechazar algo de calor hacia algún depósito que se encuentre a baja temperatura con la finalidad de completar su ciclo de trabajo. Es decir, ninguna maquina térmica puede convertir todo el calor que recibe en trabajo útil. Esta limitación de la eficiencia térmica de las maquinas térmicas forma la base para el enunciado de Kelvin-Planck de la segunda ley de la termodinámica, que se expresa como sigue: "Es imposible que un dispositivo que opera en un ciclo reciba calor de un solo depósito y produzca una cantidad neta de trabajo".

\section{Sistema empleado para la toma de mediciones}

Como se observa en Fig. 2 se utilizaron en total seis sensores de temperatura LM35 y dos DHT22 a través del circuito de refrigeración. La ubicación de cada uno de estos sensores se determinó de manera que correspondieran a puntos estratégicos del funcionamiento del sistema. También se utilizó un sensor de corriente ACS-712 que se aprovechó para tomar una medida del consumo eléctrico del equipo.

El sensor número 1 se colocó a la entrada del compresor y el 2 a la salida de este, para medir la temperatura de ingreso y salida del refrigerante en esta etapa del ciclo. Los 
sensores 3 y 4 , en la entrada y salida de aire del condensador para medir la temperatura de ingreso del aire ambiente exterior y la salida de este. El sensor 5 a la entrada de la válvula de expansión en el lugar de alta presión, y el 6 a la salida del evaporador, zona de baja presión para medir nuevamente las temperaturas del refrigerante. Los sensores 7 y 8 se utilizaron para medir la temperatura y humedad del aire ambiente interior en la entrada y salida del evaporador.

Debido a la necesidad de mantener un equilibrio térmico entre los sensores y el equipo, y para reducir pérdidas así como errores de medición, se fabricó un acople como se observa en Fig. 3, abarcando todos los sensores de temperatura para el gas refrigerante en las diferentes etapas del proceso. El acople consistió en un tubo de cobre de $1 / 2$ pulgada $(12.7 \mathrm{~mm})$ de diámetro y de 50 milímetros de largo prensado en uno de sus extremos para contener el sensor en su interior, una abrazadera de chapa de cobre de 0,8 milímetros de espesor cortada con las dimensiones necesarias para fijar el tubo a los caños del circuito de refrigeración mediante tornillos, arandelas y tuercas.

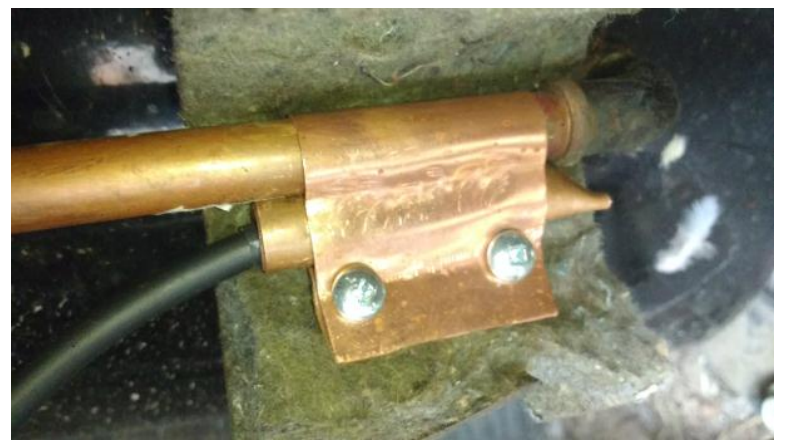

Fig. 3. Acoples utilizados para la colocación de los sensores LM35 en el circuito de refrigeración.

Una vez que se fijó el acople y se corroboró su correcta posición para la medición, se cubrió a todo el conjunto con aislante de poliestireno y se lo envolvió con cinta de refrigeración para que quede los más aislado térmicamente del aire del ambiente en sus proximidades.

Para conectar de manera provisoria los sensores a la placa de Arduino se utilizó cable tipo taller de 3 por $1,5 \mathrm{~mm}^{2}$, de diferentes medidas de largo según su ubicación en el circuito de refrigeración. Se empleó en la conexión de los sensores 1, 2, 3 y 4 un largo total del cable de 6 metros, para los 5, 6 y 7 un largo de 4 metros, y para el sensor 8 se necesitaron 10 metros de cable.

Como la mayoría de los circuitos integrados, el dispositivo LM35 tiene una capacidad limitada para manejar cargas capacitivas grandes. Este sensor solo es capaz de conducir 50 picoFaradios sin precauciones especiales. La tolerancia de la capacidad se pudo mejorar con una resistencia de 100 ohms en serie amortiguando su salida a tierra.

Sin embargo, como con cualquier circuito lineal conectado a los cables en un ambiente hostil, el rendimiento se vio afectado negativamente por fuentes electromagnéticas intensas (tales como relés, transmisores de radio, motores con cepillos, con la formación de arcos y transitorios), porque el cableado actúa como una antena de recepción y las uniones internas actúan como rectificadores. Para obtener buenas mediciones se conectó al sensor, un condensador de derivación de 10 nanoFaradios de alimentación a tierra $y$ en serie una resistencia amortiguadora, de 75 ohms en serie con 1 microFaradios para la salida a tierra, siguiendo el esquema mostrado en Fig. 4. Mediante este procedimiento se logró estabilizar los valores de las mediciones, y se observaron las mismas mediciones que en pruebas sin estos cables largos.

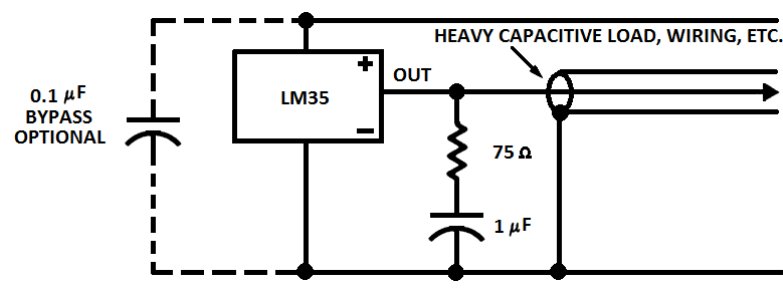

Fig. 4. Red amortiguadora RC.

\section{Visualización de las mediciones}

Para visualizar las mediciones se utilizó un programa basado en la aplicación de Parallax PLX-DAQ (Parallax Data Acquisition Tool) que fue diseñada para realizar la comunicación de datos entre los microcontroladores que fabrica esta empresa y una macro en Microsoft Excel proporcionada por esta misma.

Con esta herramienta adaptada a la plataforma de Arduino mediante la programación se logró ir tabulando los datos en una planilla de Excel, tomando el tiempo de cada medición con la hora de la $\mathrm{PC}$, un número de identificación para cada muestra, las temperaturas en grados Celsius, la humedad relativa en porcentaje, la presión del gas en el condensador en Psi, la corriente en Amperes y la potencia en Watts.

La adaptación entre el sketch de Arduino y la planilla de Excel se realizó mediante una combinación de comandos de los lenguajes de programación de cada uno, que mediante la macro de Parallax PLX-DAQ permitió la carga de los datos del monitor serial de Arduino IDE en las filas y columnas correspondientes a la tabulación en cada medición.

El programa consistió en crear distintas etiquetas en celdas consecutivas para identificar cada uno de los datos que se iban tabulando, por lo tanto, en la primera fila en la planilla de Excel se ubicó un encabezado para el dato que se iba escribiendo, siguiendo un orden para poder identificar cada valor. Aparte de los datos de las mediciones también se tabuló el tiempo de cada medición y la presión de alta del gas refrigerante. Cabe destacar que los nombres de cada una de las etiquetas se fueron cambiando durante las mediciones, para hacer más fácil su reconocimiento y uso en los cálculos posteriores.

El procedimiento para la toma de datos nos permitió ejecutar la macro en Excel de PLX-DAQ, y luego así generar una planilla nueva en blanco.

Con un monitor que establece el control de comunicación con el monitor serial de Arduino IDE, dentro de las opciones del control se colocó la tasa de comunicación 9600 baudios que siempre debe coincidir con la que se establece en el programa, así como también con el puerto de conexión.

Iniciada la comunicación se arranca tabulando los datos, en la primera fila las etiquetas de cada medición y luego los valores medidos en cada columna correspondiente. De esta manera, luego de cada muestra, estas se van ubicando en las filas siguientes según el tiempo de adquisición programado y de toma de muestras de los sensores. 
Las tomas de datos se realizaron acorde a las necesidades de estudio. Hubo momentos en los que solo se midió la temperatura en las diferentes etapas del circuito, y otros en que se hacía lo mismo, pero con la entrada y salida de aire ambiente del evaporador o la humedad.

Las mediciones de corriente y potencia se hicieron por separado según qué era lo que se iba a estudiar. Por ejemplo, para el cálculo de consumo de energía del equipo se conectaba primero el sensor de corriente en serie al compresor, luego al condensador y después al evaporador. Se midió de esta manera para obtener cada muestra por separado y en diferentes momentos, y así analizar cada una de las partes en funcionamiento. Cuando se determinó la correcta medición de cada etapa se procedió al cálculo del consumo total del equipo.

\section{E. Presión de saturación del gas Freón R-22}

Una de las magnitudes a medir fue la presión de saturación del gas refrigerante Freón R-22. La presión de vapor o más comúnmente de saturación es aquella a la que a cada temperatura las fases líquida y vapor se encuentran en equilibrio, su valor es independiente de las cantidades de líquido y vapor presentes mientras existan ambas. En la situación de equilibrio, las fases reciben la denominación de líquido y vapor saturados.

Inicialmente se buscó adaptar algún sensor comercial para este fin, pero surgieron diversos inconvenientes. Uno de los inconvenientes fue el de encontrar un sensor adecuado para medir en rangos de presión elevados que van de los 190 a los 450 Psi, y otro el de los elevados costos de este tipo de sensores. Los sensores que se estudiaron para su adquisición no se corresponden a los disponibles en la plataforma de Arduino, sino que se comercializan para equipos industriales y requerían de alguna adaptación. Dados todos estos inconvenientes se buscó un método para la medición de las altas presiones del gas refrigerante de manera indirecta. Además, se encontró que existe una relación entre la temperatura y la presión de saturación del gas R-22 y esta es provista en tablas de fabricantes de refrigerantes.

Aprovechando esta propiedad se tabularon los valores de temperatura en grados Celsius y la presión manométrica de saturación. Luego se calculó la temperatura absoluta en grados Kelvin, la presión absoluta de saturación en Psi (libras/pulgadas ${ }^{2}$ ) y la presión absoluta de saturación en kPascales haciendo uso de las herramientas estadísticas y las facilidades que nos proveyó una planilla que se creó con estos datos en Microsoft Excel.

De esta manera calculamos la presión de saturación del gas midiendo la temperatura de salida del aire recalentado del condensador a la presión de saturación del gas en esta etapa del circuito. Con los valores calculados se trazó la gráfica de presión de saturación del gas, Fig. 5, donde la temperatura en grados Celsius son las abscisas y la presión (abs) de saturación en Psi son las ordenadas. Para establecer la relación matemática que vincula la temperatura en grados Celsius y la presión (abs) de saturación del gas en Psi se trazó una línea de tendencia sobre la gráfica y se obtuvo la ecuación más conveniente para relacionar los datos. Esta ecuación (1), es polinómica de grado 3 y el coeficiente de determinación (regresión) es $\mathrm{R}^{2}=0,9998$.

$$
Y=0,0002 x^{3}+0,0292 x^{2}+2,3311 x+71.908
$$

Con la ayuda de la ecuación se pudo calcular los valores de presión de saturación del gas Freón R-22 en Psi (abs), habiendo medido solamente la temperatura en grados Celsius en la salida del aire del condensador.

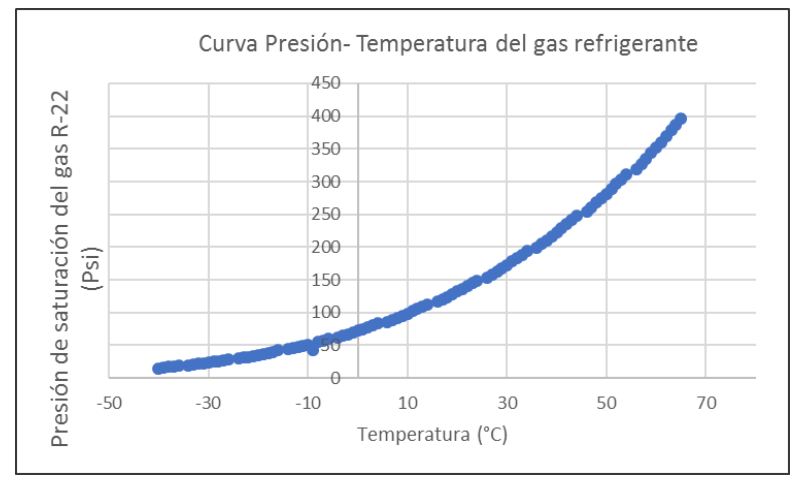

Fig. 5. Presión de saturación del gas Freón R-22 en función de la temperatura.

\section{F. Coeficiente de desempeño}

La eficiencia de un circuito de refrigeración se expresa en términos del coeficiente de desempeño (COP: siglas de coefficient of performance), el cual se denota mediante COPr. El objetivo de un circuito de refrigeración es eliminar calor (QL) del espacio refrigerado. Para lograr este objetivo se requiere la entrada de trabajo (Wnetoentrada $=$ Wne), entonces el COP de un circuito de refrigeración se puede expresar como:

$$
\begin{gathered}
C O P r=\text { Salida deseada / Entrada Requerida } \\
=Q L / W n e
\end{gathered}
$$

Esta relación también se puede expresar en forma de tasa reemplazando Wnetoentrada por el principio de conservación de la energía de un dispositivo cíclico, que requiere:

$$
\text { Wnetoentrada }=Q H-Q L
$$

Donde QL representa la magnitud del calor eliminado del espacio refrigerado, y $\mathrm{QH}$ representa la magnitud de la transferencia de calor entre el dispositivo cíclico y el medio de alta temperatura.

Entonces, la relación del COP se convierte en:

$$
C O P r=Q L /(Q H-Q L)=1 /(Q H / Q L-1)
$$

Debe de observarse que el COPr puede ser mayor que la unidad. Es decir, la capacidad de calor eliminada del espacio refrigerado puede ser mayor que la cantidad de entrada de trabajo. Esto contrasta la eficiencia térmica, la cual nunca puede ser mayor que la unidad. Una razón para expresar la eficiencia de un circuito de refrigeración con un término diferente al de máquinas térmicas, es el deseo de evitar confusiones.

\section{ENSAYOS Y RESULTADOS}

Con las mediciones realizadas, se procedió al establecimiento del ciclo de trabajo del equipo, este proceso se pudo llevar a cabo de dos maneras, uno manual mediante el uso de las curvas Presión (P) - Entalpía (h) del gas refrigerante Freón R-22 ya trazadas y que se encuentran disponibles, y otra con la ayuda del programa de simulación MollierChart.

El método manual consistió en tomar los valores medidos por los sensores para luego ir marcando los puntos de los estados del ciclo en una gráfica con las curvas P-h del 
gas refrigerante, de esta manera se pudo observar y analizar las características de los ciclos en cada medición. Con los puntos marcados y los ciclos trazados se pudo medir los valores de entalpía correspondientes, necesarios para calcular el coeficiente de eficiencia del circuito de refrigeración.

De otra manera se procedió al utilizar el programa MollierChart, Fig. 6, en este caso se introdujeron las mediciones en el simulador y este se encargó de graficar las curvas P-h del refrigerante y de trazar el ciclo de trabajo. Luego se pudo medir la entalpía ubicando el cursor sobre los puntos correspondientes.

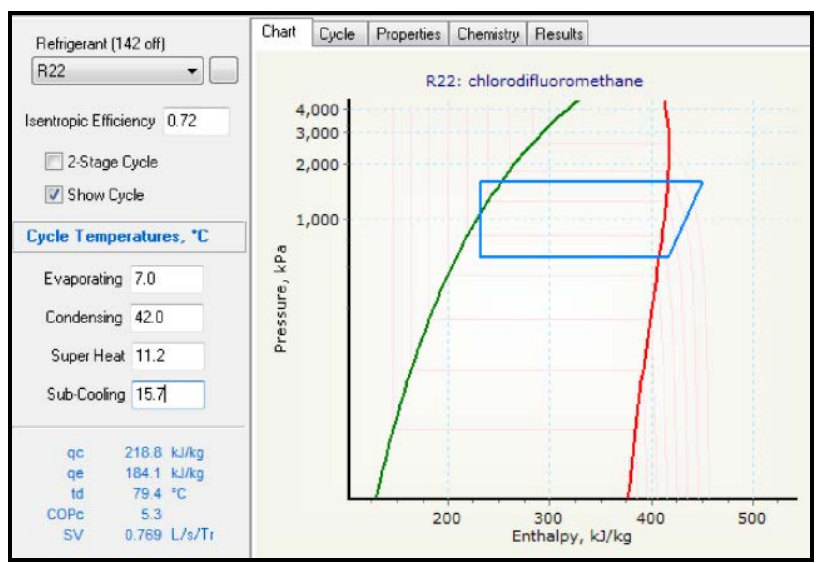

Fig. 6. Ciclo de trabajo trazado en el software en Mollier chart, con los datos obtenidos en las mediciones realizadas.

\section{G. Medición y establecimiento de un ciclo de trabajo}

Para establecer el ciclo de trabajo del equipo se utilizó una de las mediciones tabuladas, como se puede apreciar en la siguiente tabla:

TABLA II

Datos Obtenidos

\begin{tabular}{|c|c|c|}
\hline T1 & 22,48 & temperatura en ${ }^{\circ} \mathrm{C}$ en la entrada del compresor. \\
\hline $\mathbf{T 2}$ & 77,71 & temperatura en ${ }^{\circ} \mathrm{C}$ a la salida del compresor. \\
\hline T3 & 34,21 & $\begin{array}{l}\text { temperatura en }{ }^{\circ} \mathrm{C} \text { de la toma de aire ambiente } \\
\text { exterior del condensador. }\end{array}$ \\
\hline T4 & 46,2 & $\begin{array}{l}\text { temperatura en }{ }^{\circ} \mathrm{C} \text { a la salida de aire recalentado } \\
\text { del condensador. }\end{array}$ \\
\hline T5 & 30,79 & temperatura en ${ }^{\circ} \mathrm{C}$ a la entrada del evaporador. \\
\hline T6 & 11,24 & temperatura en ${ }^{\circ} \mathrm{C}$ a la salida del evaporador. \\
\hline T7 & 22,7 & $\begin{array}{l}\text { humedad relativa del ambiente en } \% \text { a la entrada de } \\
\text { aire del evaporador. }\end{array}$ \\
\hline $\mathbf{H 7}$ & 45,5 & $\begin{array}{l}\text { temperatura en }{ }^{\circ} \mathrm{C} \text { en la salida de aire del } \\
\text { evaporador. }\end{array}$ \\
\hline T8 & 15,22 & $\begin{array}{l}\text { humedad relativa \% a la salida de aire del } \\
\text { evaporador. }\end{array}$ \\
\hline H8 & 90,11 & $\begin{array}{l}\text { humedad relativa } \% \text { a la salida de aire del } \\
\text { evaporador. }\end{array}$ \\
\hline P4 & 263 & $\begin{array}{l}\text { presión de saturación del gas refrigerante en } \\
\text { Psi(abs). }\end{array}$ \\
\hline
\end{tabular}

Estas mediciones luego las cargamos en el simulador MollierChart, y después se pueden extraer los valores de entalpia necesarios para el cálculo del coeficiente de desempeño, Fig. 7.

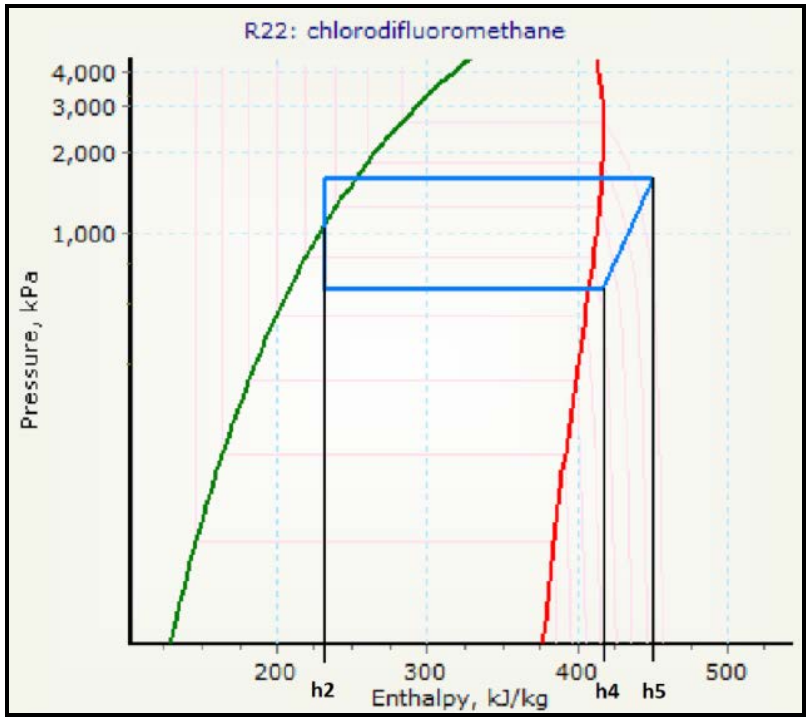

Fig. 7. Ciclo de trabajo trazado en el software en Mollier chart, con los datos obtenidos en las mediciones realizadas.

Con los cálculos realizados se pudo obtener el coeficiente de desempeño y este se comparó con el arrojado por el simulador. Además, observamos que ambos valores eran muy próximos entre sí.

$$
\begin{gathered}
Q L=h 4-h 2=415,2-232,2=183[\mathrm{Kj} / \mathrm{Kg}] \\
W=h 5-h 4=450,1-415,2=34,9[\mathrm{Kj} / \mathrm{Kg}] \\
C O P r=Q L / W=183 / 34,9=5,24
\end{gathered}
$$

\section{H. Consumo de energía eléctrica del equipo}

Se pudo determinar el consumo eléctrico del equipo en funcionamiento. Para esto se midió la corriente en diferentes momentos y en diferentes lugares del circuito. Se calculó la potencia en kWatts de cada una de ellas y luego la potencia total consumida.

TABLA III

Mediciones Realizadas

\begin{tabular}{|l|l|l|}
\hline I1 & 0,65 & $\begin{array}{l}\text { Corriente eficaz en Amperes medida en el } \\
\text { condensador. }\end{array}$ \\
\hline $\mathbf{I 2}$ & 10,43 & $\begin{array}{l}\text { Corriente eficaz en Amperes medida en el } \\
\text { compresor. }\end{array}$ \\
\hline $\mathbf{I 3}$ & 0,43 & $\begin{array}{l}\text { corriente eficaz en Amperes medida en el } \\
\text { evaporador. }\end{array}$ \\
\hline Itotal & 11,51 & Corriente total en Amperes que se calculó. \\
\hline W1 & 0,15 & Potencia en kWatts del condensador. \\
\hline W2 & 2,4 & Potencia en kWatts del compresor. \\
\hline W3 & 0,1 & Potencia en kWatss del evaporador. \\
\hline Wtotal & 2,65 & Potencia total en kWatts que se calculó. \\
\hline & & \\
\hline
\end{tabular}

Donde, Itotal se refiere a la corriente que resulta de la adición de I1, I2 e I3. Y Wtotal se refiere a la potencia total consumida por el equipo, que resulta de la adición de W1, W2 y W3.

Para contrastar estos datos también se midió la corriente en cada caso con una pinza amperométrica y un wattimetro, y se observó una muy buena aproximación entre ambos resultados. 


\section{Análisis del funcionamiento}

Para analizar el funcionamiento del equipo desde el encendido hasta pasado un cierto tiempo en el cual se estabiliza el ciclo, se tomaron mediciones a lo largo de este período y se graficó para establecer un análisis de las curvas. La observación de estos gráficos permitió la identificación de los estados en cada parte del ciclo.

Se observó en cada serie de muestras el aumento y disminución de la temperatura del refrigerante en cada etapa del circuito, la variación de la temperatura del aire y de la humedad relativa, tanto en el ambiente exterior donde está el condensador, como en el ambiente interior donde se ubica el evaporador. También se midió la presión de saturación del gas en el condensador, utilizando la ecuación polinómica para relacionar la temperatura del aire a la salida del condensador y esta.

\section{J. Establecimiento del ciclo de trabajo mediante las curvas $P$-h del gas refrigerante Freón $R-22$}

En este caso se trató el ciclo de trabajo mediante las curvas P-h del refrigerante, el trabajo consistió en trazar las zonas de funcionamiento en base a las mediciones tomadas, y en un gráfico ya realizado con el diagrama de Mollier del gas se iban marcando las diferentes etapas.

Se inició con la lectura de las mediciones de todos los sensores y para la presión baja de funcionamiento del equipo, se determinó que esta se podía suponer como constante durante este proceso debido a su poca variación respecto a la presión del lado de alta para su trazado en las curvas.

El proceso consistió en observar la presión manométrica y luego con este valor se trazó una línea recta marcando la isobara correspondiente en el diagrama.

Una vez que se finalizó con el tratamiento de la presión de baja del equipo se tomó lectura de la medición de la presión de alta para ingresar en el gráfico, y después mediante este valor se trazó la línea recta paralela al eje de las abscisas que representa esta isobara.

Cuando finalizamos el trazado de las rectas se ubicó en el gráfico los puntos pertenecientes al sobrecalentamiento y subenfriamiento medidos con los sensores. Para esto se marcó un primer punto perteneciente a la temperatura de ingreso del refrigerante al compresor. Después se indicó un segundo punto que establecía la temperatura del refrigerante a la salida del compresor.

Con la temperatura a la entrada del evaporador se marcó un tercer punto, y con la medición de la temperatura del condensador un cuarto.

Para marcar un quinto punto se trazó una línea recta paralela al eje de las ordenadas uniendo el quinto punto con la recta de baja presión. Un sexto punto es el cruce de la recta de baja presión con la curva de $100 \%$ gas.

Al terminar este proceso se obtuvo como resultado el trazado completo del ciclo de trabajo del equipo de enfriamiento utilizando las mediciones de los sensores y las curvas P-h del gas Freón R-22.

Mediante este trabajo se observó que durante el ciclo hubo un sobrecalentamiento de $12^{\circ} \mathrm{C}$ y un subenfriamiento de $10,1^{\circ} \mathrm{C}$.
Se calculó el coeficiente de desempeño en base a los valores de entalpías marcados.

\section{CONCLUSIONES}

Se implementó un método para la calibración de sensores y puesta a punto de sus conexiones en el circuito de refrigeración. Se pudo establecer un procedimiento para la tabulación de las mediciones, mediante una planilla de Excel utilizando el sistema basado en la plataforma Arduino. Con las mediciones realizadas, se calculó de manera indirecta la presión de saturación del gas refrigerante Freón R-22 y se utilizó para el trazado del ciclo en las curvas P-h. Se logró medir y analizar el consumo de energía eléctrica del equipo de enfriamiento durante su funcionamiento. Mediante los datos obtenidos durante las diferentes mediciones, se trabajó con varios métodos para el establecimiento del ciclo de trabajo y se logró calcular el coeficiente de desempeño, contrastando con otras herramientas los resultados.

El método conlleva las ventajas de cumplir con fines didácticos y para establecer una relación con los contenidos desarrollados durante las clases del laboratorio de Termodinámica. También se puede resaltar que fue realizado con un bajo costo y este no requirió de un gran presupuesto.

Para mejorar el método sería muy bueno lograr agregar un sensor para la captura de las presiones en los puntos estratégicos del circuito de refrigeración y que estos se puedan ir tabulando en conjunto a las temperaturas y los valores de humedad. Si se lograra este agregado se podría, además de establecer el ciclo de trabajo del equipo, automatizar su funcionamiento para lograr mantener un consumo y rendimientos óptimos de manera permanente.

\section{AGRADECIMIENTOS}

Federico Roffé reconoce el apoyo de la Agencia Nacional de Promoción Científica y Tecnológica a través del PICT 2016-1056.

\section{REFERENCIAS}

[1] Yonus A. Cengel - Michael A. Boles - Termodinámica - 4ta Edición.

[2] M.M. Dominguez - A. J. Rovira de Antonio - Ed. Digital Maquinas Térmicas - marzo 1994.

[3] José Echegaray - Tratado elemental de termodinámica - 1868.

[4] United Nations Eviroments Programme and Ozone Secretariat. Montreal Protocol on Substances that Deplete the Ozone Layer as either adjusted and/or amended in London 1990, Copenhagen 1992, Vienna 1995, Montreal 1997, Beijing 1999. Nairobi, Kenya: Ozone Secretariat, United Nations Environment Programme, 2000. ISBN 92807188869789280718881.

[5] Ei-Awad, M. M. Validation of computerized analytical model for evaluating natural hydrocarbon mixtures as alternative refrigerants. Journal of Sustainable Energy and Environment. 2011. Vol. 2, p. 175-179.

[6] Y. A. Cengel - Termodinamica - Capitulo 6 - sexta edicion.

[7] American Society of Heating, Refrigeration and Air-Conditioning Engineers, Handbook of fundamentals, Atlanta: ASHRAE 2017.

[8] E. W. Lemmon, M. L. Huber, M. O. McLinden, 2007, NIST Reference Fluid Thermodynamic and Transport PropertiesREFPROP Version 8.0, User's Guide, National Institute of Standards and Technology, Physical and Chemical Properties Division, Boulder, Colorado 80305. 